\title{
Mean-field solution of the small-world network model
}

\author{
M. E. J. Newman, C. Moore, and D. J. Watts \\ Santa Fe Institute, 1399 Hyde Park Road, Santa Fe, NM 87501
}

\begin{abstract}
The small-world network model is a simple model of the structure of social networks, which simultaneously possesses characteristics of both regular lattices and random graphs. The model consists of a one-dimensional lattice with a low density of shortcuts added between randomly selected pairs of points. These shortcuts greatly reduce the typical path length between any two points on the lattice. We present a mean-field solution for the average path length and for the distribution of path lengths in the model. This solution is exact in the limit of large system size and either large or small number of shortcuts.
\end{abstract}


Social networks, such as networks of friends, have two characteristics which one might imagine were contradictory. First, they show "clustering," meaning that two of your friends are far more likely also to be friends of one another than two people chosen from the population at random. Second, they exhibit what has become known as the "small-world effect," namely that any two people can establish contact by going through only a short chain of intermediate acquaintances. Following the work of Milgram [1], it is widely touted that the average number of such intermediates is about six-there are "six degrees of separation" between two randomly chosen people in the world. In fact this number is probably not a very accurate estimate, but the basic principle is sound.

These two properties appear contradictory because the first is a typical property of lowdimensional lattices but not of random graphs or other high-dimensional lattices, while the second is typical of random graphs, but not of low-dimensional lattices. Recently, Watts and Strogatz [2] have proposed a simple model of social networks which interpolates between lowdimensional lattices and random graphs and displays both the clustering and small-world properties. In this model, $L$ sites are placed on a regular one-dimensional lattice with nearest- and next-nearest-neighbor connections out to some constant range $k$ and periodic boundary conditions (the lattice is a ring). A number of shortcuts are then added between randomly chosen pairs of sites with probability $\phi$ per connection on the underlying lattice (of which there are $L k$ ). Thus there are on average $L k \phi$ shortcuts in the graph. An example of a small-world graph with $L=24, k=3$, and four shortcuts is shown in Fig. 11a. Watts and Strogatz examined numerically the average distance between pairs of vertices on smallworld graphs and found that only a small density of shortcuts is needed to produce distances comparable to those seen in true random graphs. At the same time, the model shows the clustering which is characteristic of real social networks.

In this paper we derive an analytic solution for the distribution of path lengths in the small-world model. To do this we make use of a mean-field approximation in which the distributions of quantities over the randomness are represented by the average values of those quantities. However, as we will show, the mean-field theory turns out to be exact in 

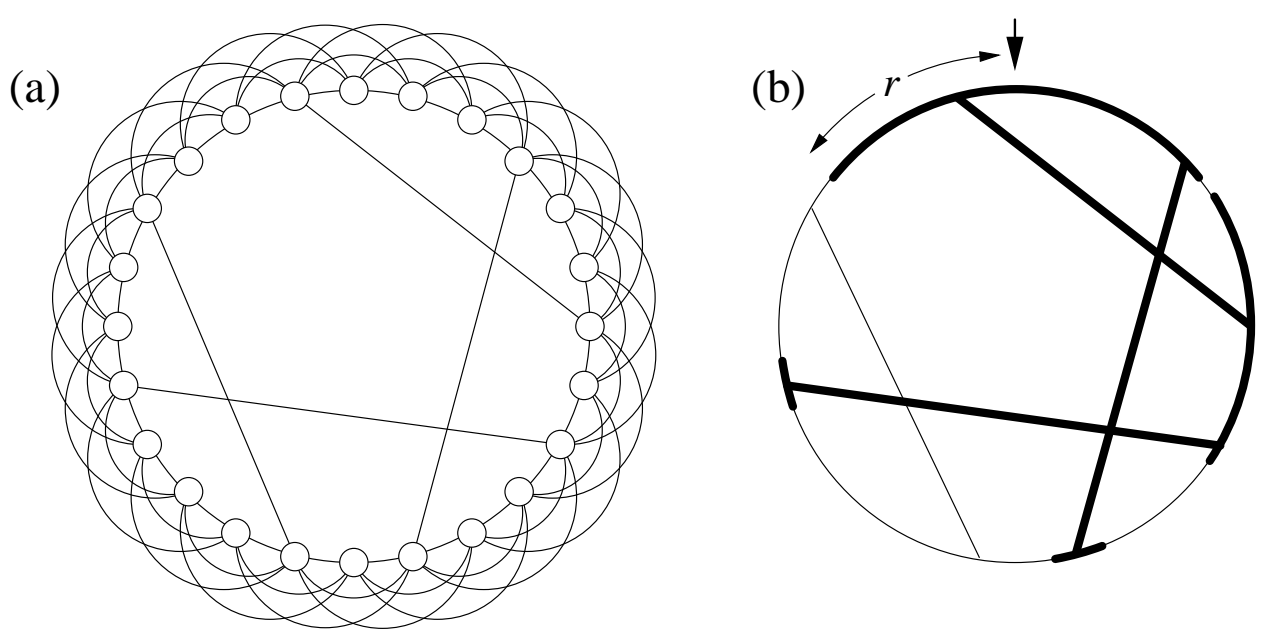

Figure 1 (a) A small-world graph of 24 sites with $k=3$ and four shortcuts. (b) The continuum version of the same graph. The bold lines denote the portion of the graph which is within distance $r$ of the point at the top denoted by the arrow. In this case there are four filled segments, or "clusters", around the perimeter of the graph, or equivalently four gaps between clusters.

the limit of large system size for the small-world model.

The approach we use is first to solve the continuum version of the Watts-Strogatz model shown in Fig. 1 $\mathrm{b}$. In this version of the model the underlying one-dimensional lattice is treated as a continuum, and one can measure the distance between any two points on this continuum, rather than between only a discrete set of lattice sites. Shortcuts are assumed to have length zero. Once we have a solution for the continuum model, we then note that if the density of shortcuts is low the discrete and continuum models are equivalent, and hence our solution is also a solution of the discrete small-world model.

Consider then a "neighborhood" of radius $r$ centered around a randomly chosen point on a small-world network of $L$ sites, where by neighborhood we mean the set of points which can be reached by following paths of length $r$ or less on the graph. Let $m(r)$ be the number of sites on the graph which do not belong to this neighborhood, averaged over many realizations of the randomness in the graph, and $n(r)$ be the average number of "gaps" around the lattice amongst which those sites are divided-see Fig. 1 b. Equivalently, $n(r)$ 
can be viewed as the number of "clusters" of occupied sites. In the continuum model both $m$ and $n$ are real numbers. We will also find it convenient to use the rescaled variables

$$
\mu(r)=\frac{m(r)}{L}, \quad \nu(r)=\frac{n(r)}{L} .
$$

In the continuum limit the quantities $m(r)$ and $n(r)$ satisfy differential equations as follows. The rate at which the number of empty sites on the lattice decreases with increasing $r$ is equal to the number $2 n$ of growing edges of clusters on the lattice times the range $k$ of connections on the lattice. Thus

$$
\frac{\mathrm{d} m}{\mathrm{~d} r}=-2 k n
$$

or

$$
\frac{\mathrm{d} \mu}{\mathrm{d} r}=-2 k \nu
$$

This equation is exact for all values of $L$ and $\phi$.

The rate at which the number of gaps $n$ changes has two contributions. First, the number of gaps increases as a result of the shortcuts on the graph. If $\xi=1 /(k \phi)$ is the characteristic length defined in Ref. [3] such that $L / \xi$ is the average number of shortcuts in the graph, then the density of the ends of shortcuts on the lattice is $2 / \xi$. This means that as $r$ increases, new shortcuts are encountered at a rate $4 k n / \xi$. For each shortcut encountered, a new cluster will be started at a random position on the lattice, provided that the other end of the shortcut in question falls in one of the gaps around the ring. The probability of this happening is $m / L$. Thus the rate at which clusters (or gaps) are created is $4 k m n / \xi L$.

The number of gaps decreases when the edges of a gap meet one another. This will happen in the interval from $r$ to $r+\delta r$ if the size of one of the gaps is less than $2 k \delta r$. If we consider all possible ways of distributing the $m$ empty sites over $n$ gaps, we can see that the probability distribution of the sizes of the gaps is the same as the distribution of the smallest of $n-1$ uniformly distributed random numbers $x$ between 0 and $m$, which is

$$
p(x)=\frac{n-1}{m}\left[1-\frac{x}{m}\right]^{n-2} .
$$


Thus the probability of one particular gap being smaller than $2 k \delta r$ is $1-(1-2 k \delta r / m)^{n-1}$, which tends to $2 k(n-1) \delta r / m$ in the limit of small $\delta r$. The probability that any one of them is smaller than $2 k \delta r$ is $n$ times this. Thus our final equation for the rate of change of $n$ is

$$
\frac{\mathrm{d} n}{\mathrm{~d} r}=\frac{4 k m n}{\xi L}-\frac{2 k n(n-1)}{m},
$$

or

$$
\frac{\mathrm{d} \nu}{\mathrm{d} r}=\frac{4 k \mu \nu}{\xi}-\frac{2 k \nu(\nu-1 / L)}{\mu}
$$

This equation is only exact when the average values $\mu(r)$ and $\nu(r)$ accurately represent the actual values of these quantities in the particular realization of the model we are looking at, i.e., when the distribution of values is sharply peaked. This will be the case when the number of shortcuts on the lattice is either much less than one $L \ll \xi$ - or much greater than one $-L \gg \xi$-and therefore also in the limit of large system size. We have confirmed this using numerical simulations, which show the distributions of $\mu$ and $\nu$ to be sharply peaked in these limits but broad elsewhere.

Between them, Eqs. (3) and (6) are the fundamental equations which lead to our solution for the small-world model. As demonstrated in the appendix, these equations can also be derived by writing down difference equations for the variables $m$ and $n$ in the discrete smallworld model and then expanding in powers of the shortcut density $\phi=1 / \xi$ and keeping only the leading order terms.

We solve Eqs. (3) and (6) as follows. First, we take their ratio, which eliminates the variable $r$ and gives us a single differential equation directly relating $\mu$ and $\nu$ thus:

$$
\frac{\mathrm{d} \nu}{\mathrm{d} \mu}=-\frac{2 \mu}{\xi}+\frac{\nu-1 / L}{\mu} .
$$

The general solution of this equation is

$$
\nu=-\frac{2 \mu^{2}}{\xi}+\frac{1}{L}+C \mu
$$

where $C$ is an integration constant. The constant can be fixed using the boundary conditions $\mu(0)=1, \nu(0)=1 / L$, which imply that $C=2 / \xi$ and hence 


$$
\nu=\frac{2}{\xi}\left(\mu-\mu^{2}\right)+\frac{1}{L}
$$

Substituting this solution back into Eq. (3), we get

$$
\frac{\mathrm{d} \mu}{\mathrm{d} r}=\frac{4 k}{\xi}\left(\mu^{2}-\mu\right)+\frac{2 k}{L} .
$$

If we neglect the constant term in this equation, we arrive at the normal logistic growth equation [4], which will give an accurate solution for $\mu$ in the regime where the lattice is neither very full nor very empty. If we keep all the terms, the general solution given the boundary conditions is

$$
\begin{aligned}
r & =\frac{\xi}{4 k} \int_{1}^{\mu} \frac{\mathrm{d} z}{z^{2}-z-\xi / 2 L} \\
& =\frac{\xi}{2 k \sqrt{1+2 \xi / L}}\left[\tanh ^{-1} \frac{1}{\sqrt{1+2 \xi / L}}-\tanh ^{-1} \frac{2 \mu-1}{\sqrt{1+2 \xi / L}}\right] .
\end{aligned}
$$

Rearranging for $\mu$ this gives

$$
\mu=\frac{1}{2}\left[1+\sqrt{1+2 \xi / L} \tanh \left(\tanh ^{-1} \frac{1}{\sqrt{1+2 \xi / L}}-2 \sqrt{1+2 \xi / L} \frac{k r}{\xi}\right)\right] .
$$

This equation gives us $\mu$ in terms of $r, \xi$ and $L$ for the continuum version of the small-world model. In the case where the typical lattice distance between the ends of shortcuts is much larger than one $-\xi \gg 1$ - the continuum version becomes equivalent to the normal discrete version of the model and so in this limit our solution is also a solution of the discrete smallworld model. Combining this condition with the conditions specified earlier, we see that our solution will be exact when either $1 \ll L \ll \xi$, or when $1 \ll \xi \ll L$. This latter regime is precisely the regime in which the small-world model is physically interesting: the regime of large system size and large number of sparsely distributed shortcuts. In the intermediate regime between the two conditions given, the solution is still quite accurate, and gives a good guide to the general behavior of the model, as we will shortly show.

We now derive some of the more important consequences of Eq. (12). First, we check that it reduces to the correct expression in the case $L \rightarrow \infty$. Making use of the identity

$$
\tanh \left(x_{1}+x_{2}\right)=\frac{\tanh x_{1}+\tanh x_{2}}{1+\tanh x_{1} \tanh x_{2}}
$$


and we find that to first order in $\xi / L$

$$
\mu=1+\frac{\xi}{2 L}\left[1-\mathrm{e}^{4 k r / \xi}\right]
$$

which agrees with the direct derivation for the $L=\infty$ case in Ref. [3].

Next, we note that once we have the fraction $\mu$ of sites not belonging to a neighborhood of radius $r$, we can also calculate the number $A(r) \mathrm{d} r$ in an interval from $r$ to $r+\mathrm{d} r$ - the "surface area" of the neighborhood-from

$$
A=-L \frac{\mathrm{d} \mu}{\mathrm{d} r}=2+\frac{4 L}{\xi}\left(\mu-\mu^{2}\right)
$$

Thus, once we have $\mu$ we can easily calculate $A$.

We can also derive an expression for the average vertex-vertex separation $\ell$ on the graph, a quantity which has been studied by many authors [2, 3,5] - We write

$$
\ell=\frac{1}{L} \int_{0}^{\infty} r A(r) \mathrm{d} r=\int_{0}^{1} r \mathrm{~d} \mu,
$$

where we have made use of Eq. (15). Even before performing the integral, we can see that this implies certain behavior on the part of $\ell$. Eq. (11) shows that $k r / \xi$ is a function only of $\mu$ and of the ratio of $L$ and $\xi$. In other words $r$ has the form

$$
r=\frac{\xi}{k} h(\mu, L / \xi)
$$

where $h(x, y)$ is a universal scaling function with no dependence on the parameters of the model other than through its arguments. Substituting this form into Eq. (16) and performing the integral over $\mu$, we get

$$
\ell=\frac{\xi}{k} g(L / \xi)
$$

where $g(x)$ is another universal scaling function. Except for the leading factor of $1 / k$, this scaling form is identical to the one suggested previously by Barthélémy and Amaral [6]. Making the substitution $g(x)=x f(x)$, we can also write it in the form

$$
\ell=\frac{L}{k} f(L / \xi)=\frac{L}{k} f(L k \phi)
$$


a form which was proposed by Newman and Watts on the basis of renormalization group arguments, and which has been confirmed by extensive numerical simulation [0,8].

The complete solution for $\ell$ is obtained by substituting (11) into (16) and performing the integral, which gives

$$
\ell=\frac{\xi}{2 k \sqrt{1+2 \xi / L}} \tanh ^{-1} \frac{1}{\sqrt{1+2 \xi / L}} .
$$

The scaling function $f(x)$ is then given by

$$
f(x)=\frac{1}{2 \sqrt{x^{2}+2 x}} \tanh ^{-1} \sqrt{\frac{x}{x+2}} .
$$

In Fig. 2 we show this form for the scaling function along with numerical data from direct measurements of the average path length on $k=1$ discrete small-world graphs of size up to $L=10^{7}$ sites. As the figure shows, the two are in good agreement for large and small values of the independent variable $x$ but, as expected, there is some disagreement in the region around $x=1$ where $\xi$ and $L$ are of the same order of magnitude.

The asymptotic forms of Eq. (21) are

$$
f(x) \sim \begin{cases}\frac{1}{4} & \text { for } x \ll 1 \\ (\log 2 x) / 4 x & \text { for } x \gg 1,\end{cases}
$$

where we have made use of the identity

$$
\tanh ^{-1} x=\frac{1}{2} \log \frac{1+x}{1-x}
$$

These forms are in agreement with previous conjectures [6,7], which suggested that $f(x)$ should have the value $\frac{1}{4}$ for small $x$ and should go as $(\log x) / x$ for large $x$. As we see, the leading numerical factor in the latter case is $\frac{1}{4}$; this figure is exact, since Eq. (12) is exact for large $L / \xi$.

In passing, we note that there is a simple physical interpretation of the scaling function $f(x)$ : apart from the leading factor of $\frac{1}{4}$, it is the fraction by which the average path length on a small-world graph is reduced if the graph has $x$ shortcuts. For example, Eq. (21) indicates that it takes $x=3.5$ shortcuts on average to reduce the mean path length by a 


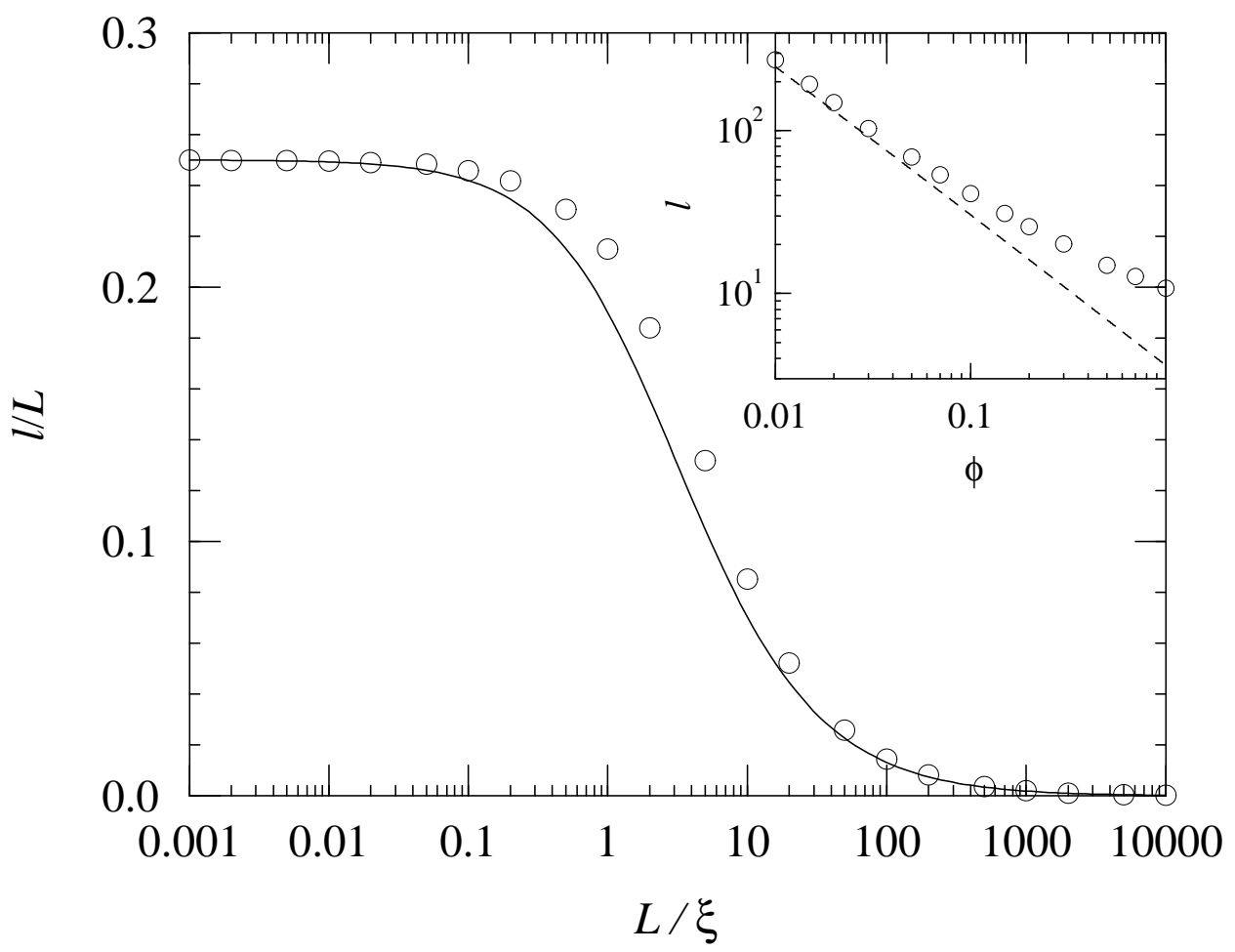

Figure 2 The average path length as a fraction of system size on a $k=1$ smallworld graph, plotted against the average number $L / \xi$ of shortcuts. The circles are numerical measurements for the discrete model and the solid line is the analytic solution for the continuum model, Eq. (21). The error bars on the numerical measurements are smaller than the points. Inset: the average path length on small-world graphs with $L=10^{6}$, for values of $\phi$ from 0.01 up to 1 (circles) and the analytic solution, Eq. 20) (dotted line).

half, and 44 shortcuts to reduce it by a factor of ten. Thus only a small number of shortcuts are needed to reduce path lengths quite considerably. The same conclusion has been reached by Watts and Strogatz [2] on the basis of numerical data.

In the inset of Fig. 2 we show how our solution fails when the shortcut density becomes too high. The figure shows numerical results for $\ell$ for a variety of values of the shortcut density $\phi=1 / \xi$ from 0.01 up to 1 , for systems of one million sites (circles). The dotted line is Eq. (20). As the figure shows, the analytic solution is a reasonable guide to the behavior of $\ell$ up to quite large values of $\phi$ but, as expected, fails when $\phi$ gets close to 1 .

To conclude, we have given a mean-field-like analytic solution for the distribution of path 
lengths in the continuum version of the Watts-Strogatz small-world model. This solution is exact in the limit of large system sizes for a given density of shortcuts, or in the limit of low shortcut density for given system size. In the case where the shortcut density is low but the total number of shortcuts on the lattice is large (because the lattice itself is also large) our solution is also an exact solution of the normal discrete small-world model. We have also derived an expression for the average path length in the model and from this extracted the scaling forms which this path length obeys. We have checked our results against numerical simulation of the discrete small-world model and find good agreement in the regions where our solution is expected to be exact. In other regions the solution is a good guide to the general behavior of the model but shows some deviation from the numerical results. 


\section{APPENDIX: THE CONTINUUM MODEL AS THE SMALL- $\phi$ LIMIT OF THE DISCRETE MODEL}

In this appendix, we rederive Equations (3) and (6) from the behavior of the discrete version of the small-world model to leading order in the shortcut density $\phi$.

Consider a neighborhood of sites which are within distance $r$ of a given starting site in the discrete model. By analogy with the continuous case, let $m$ be the number of sites on the lattice which are not in this neighborhood and $n$ be the number of "gaps" between clusters of occupied sites around the ring. In fact, in the spirit of our mean-field approximation, $m$ and $n$ should be thought of as the average of these quantities over all possible realizations of lattice. This means that they may have non-integer values. Here we treat them as integers

for combinatorial purposes, but our formulas are easily extended to non-integer values by replacing the factorials by $\Gamma$-functions.

When we increase $r$ by one, the value of $m$ decreases for two reasons: first because the gaps between clusters shrink and second because of new sites which are reached by traveling down shortcuts encountered on the previous step. We write

$$
m^{\prime}=m-\Delta m
$$

where

$$
\Delta m=\Delta m_{g}+\Delta m_{s}
$$

with the two terms representing the shrinking of gaps and the shortcut contribution respectively.

To calculate $\Delta m_{g}$, we note that the probability of any particular gap having size $j$ is

$$
p_{j}=\frac{\left(\begin{array}{c}
m-j-1 \\
n-2
\end{array}\right)}{\left(\begin{array}{c}
m-1 \\
n-1
\end{array}\right)},
$$

and the average number of such gaps is $n$ times this. When we increase $r$ by one, gaps of size $2 k$ or larger shrink by $2 k$, while gaps smaller than $2 k$ vanish altogether. Thus 


$$
\Delta m_{g}=n\left[\sum_{j=1}^{2 k} j p_{j}+2 k\left(1-\sum_{j=1}^{2 k} p_{j}\right)\right]=m-\frac{(m-2 k) !(m-n) !}{(m-1) !(m-n-2 k) !} .
$$

To calculate the contribution $\Delta m_{s}$ from the number of shortcuts, we note that the probability of encountering the end of a shortcut at any given site is $2 / \xi=2 k \phi$, just as in the continuum case, so the number of new shortcuts encountered when we increase $r$ by one is $2 k \phi \Delta m . \Delta m_{s}$ in fact depends on the number of shortcuts encountered on the previous increase in $r$, so we need to write $2 k \phi \Delta m^{(r-1)}$. Only those shortcuts which land us at one of the $m-\Delta m_{g}$ unoccupied sites contributes to $\Delta m_{s}$, so

$$
\Delta m_{s}=2 k \phi \Delta m^{(r-1)}\left[m-\Delta m_{g}^{(r)}\right]
$$

Substituting Eqs. (A4) and (A5) into Eq. (A2) we get our complete expression for $\Delta m$. Now we note that, except when the lattice is very nearly full, the number of unfilled sites $m$ is of order $L$. The number of clusters of filled sites can be no greater than the number of shortcuts on the lattice plus one for the initial cluster around the starting point. Thus $n \leq \phi L+1$, and the ratio $(n-1) / m$ is a quantity of order $\phi$. Expanding in powers of this quantity and assuming the number of sites $m$ to be much greater than $2 k$ then gives

$$
\Delta m=2 k n
$$

plus terms of order $\phi$. Physically, the reason for the simplicity of this expression is that, to first order in $(n-1) / m$, most gaps have size $2 k$ or larger, and the contribution from new shortcuts to can be neglected, since most sites are connected only to their local neighbors.

The change in the value of $n$ has also two contributions. First, the number of gaps increases when a shortcut creates a new cluster, and divides a gap into two new ones. As we have already shown this happens $\Delta m_{s}$ times on average when we increase $r$ by one, where $\Delta m_{s}$ is given by Eq. (A5). Second, gaps disappear when their edges meet. When we increase $r$ by one, a gap will close if its size is $2 k$ or less. Thus the change in $n$ is

$$
n^{\prime}=n+\Delta n
$$


where

$$
\Delta n=\Delta m_{s}-n \sum_{j=1}^{2 k} p_{j}=\Delta m_{s}-n \frac{(m-n) !(m-2 k-1) !}{(m-1) !(m-n-2 k) !}
$$

where $\Delta m_{s}$ is given by Eq. (A5). Expanding to lowest order in $(n-1) / m$, taking $m \gg 2 k$ again, and combining the result with Eq. (A5) gives

$$
\Delta n=\frac{4 k^{2} \phi m n}{L}-\frac{2 k n(n-1)}{m} .
$$

Changing Eqs. (A6) and (A9) from difference equations to differential ones and dividing by $L$ to transform from $m$ and $n$ to $\mu$ and $\nu$ gives

$$
\begin{aligned}
& \frac{d \mu}{d r}=-2 k \nu, \\
& \frac{d \nu}{d r}=4 k^{2} \phi \mu \nu+\frac{2 k \nu(\nu-1 / L)}{\mu} .
\end{aligned}
$$

Recalling that $\xi=1 /(k \phi)$, we can see that these equations are identical to Equations (3) and (6). 


\section{REFERENCES}

[1] S. Milgram, "The small world problem," Psychol. Today 2, 60-67 (1967).

[2] D. J. Watts and S. H. Strogatz, "Collective dynamics of 'small-world' networks," Nature 393, 440-442 (1998).

[3] M. E. J. Newman and D. J. Watts, "Scaling and percolation in the small-world network model," Phys. Rev. E, in press.

[4] S. H. Strogatz, Nonlinear Dynamics and Chaos, Addison-Wesley (Reading, MA).

[5] C. F. Moukarzel, "Spreading and shortest paths in systems with sparse long-range connections," submitted to Phys. Rev. E.

[6] M. Barthélémy and L. A. N. Amaral, "Small-world networks: Evidence for a crossover picture," Phys. Rev. Lett. 82, 3180-3183 (1999).

[7] M. E. J. Newman and D. J. Watts, "Renormalization group analysis of the small-world network model," Phys. Lett. A, in press.

[8] M. Argollo de Menezes, C. F. Moukarzel and T. J. P. Penna, "First-order transition in small-world networks," submitted to Phys. Rev. Lett.

[9] A. Barrat and M. Weigt, "On the properties of small-world network models," Euro. Phys. $J . B$, in press. 